\title{
Contribution of intraoperative neuromonitoring to the identification of the external branch of superior laryngeal nerve
}

\author{
Nurcihan Aygün ${ }^{1}$, Mehmet Uludağ' ${ }^{1}$, Adnan Isşgör ${ }^{2}$
}

\section{ABSTRACT}

Cite this paper as:

Aygün N, Uludağ M, İ̧gör A.

Contribution of intraoperative neuromonitoring to the identification of the external branch of superior laryngeal nerve. Turk J Surg 2017; 33: 169-174.

This study was presented at the $7^{\text {th }}$ National Endocrine Surgery Congress, 23-26 April 2015,

Antalya, Turkey.

'Department of General Surgery, Şişli Hamidiye Etfal Training and Research Hospital, İstanbul, Turkey

${ }^{2}$ Department of General Surgery, Bahçeşehir University School of Medicine, İstanbul, Turkey

Address for Correspondence Mehmet Uludağ

e-mail:drmuludag@hotmail.com

Received: 12.06 .2016

Accepted: 10.08.2016

(c) Copyright 2017

by Turkish Surgical Association

Available online at

www.turkjsurg.com
Objective: We evaluated the contribution of intraoperative neuromonitoring to the visual and functional identification of the external branch of the superior laryngeal nerve.

Material and Methods: The prospectively collected data of patients who underwent thyroid surgery with intraoperative neuromonitoring for external branch of the superior laryngeal nerve exploration were assessed retrospectively. The surface endotracheal tube-based Medtronic NIM3 intraoperative neuromonitoring device was used. The external branch of the superior laryngeal nerve function was evaluated by the cricothyroid muscle twitch. In addition, contribution of external branch of the superior laryngeal nerve to the vocal cord adduction was evaluated using electromyographic records.

Results: The study included data of 126 (female, 103; male, 23) patients undergoing thyroid surgery, with a mean age of $46.2 \pm 12.2$ years (range, 18-75 years), and 215 neck sides were assessed. Two hundred and one (93.5\%) of 215 external branch of the superior laryngeal nerves were identified, of which $60(27.9 \%)$ were identified visually before being stimulated with a monopolar stimulator probe. Eighty-nine (41.4\%) external branch of the superior laryngeal nerves were identified visually after being identified with a probe. Although $52(24.1 \%)$ external branch of the superior laryngeal nerves were identified with a probe, they were not visualized. Intraoperative neuromonitoring provided a significant contribution to visual $(p<0.001)$ and functional $(p<0.001)$ identification of external branch of the superior laryngeal nerves. Additionally, positive electromyographic responses were recorded from 160 external branch of the superior laryngeal nerves (74.4\%).

Conclusion: Intraoperative neuromonitoring provides an important contribution to visual and functional identification of external branch of the superior laryngeal nerves. We believe that it can not be predicted whether the external branch of the superior laryngeal nerve is at risk or not and the nerve is often invisible; thus, intraoperative neuromonitoring may routinely be used in superior pole dissection. Glottic electromyography response obtained via external branch of the superior laryngeal nerve stimulation provides quantifiable information in addition to the simple visualization of the cricothyroid muscle twitch.

Keywords: Thyroidectomy, intraoperative neuromonitoring, external branch of the superior laryngeal nerve, nerve identification, cricothyroid muscle twitch

\section{INTRODUCTION}

Voice impairments are common complaints after thyroidectomy. They are considered to be multifactorial in origin and can occur due to the trauma of neural or other laryngotracheal structures (1). The preservation of both the recurrent laryngeal nerve (RLN) and external branch of the superior laryngeal nerve (EBSLN) is necessary to maintain vocal cord function (2).

The superior laryngeal nerve is a branch of the vagus nerve near its exit from the jugular foramen of the skull base. It usually arises from the nodose ganglion of the vagus at the level of $C 2$, about $4 \mathrm{~cm}$ cranially to the carotid artery bifurcation. In addition, it receives a branch from the superior cervical sympathetic ganglion.

About $1.5 \mathrm{~cm}$ caudally, the superior laryngeal nerve divides into a larger internal branch and a smaller external branch. The EBSLN descends dorsally to the carotid sheath and then crosses medially towards the larynx. The EBSLN has a course dorsal to the superior thyroid artery and superficial to the inferior pharyngeal constrictor muscle as it descends and runs medially to innervate the cricothyroid muscle (СTM) (3-5).

The CTM maintains the tension of the vocal folds during the production of high-pitched sounds (3). The position of EBSLN exposes it to the risk of injury during dissection of the superior thyroid pole. CTM paralysis caused by EBSLN injury does not enable the pitch elevation, which is particularly a great problem for women and voice professionals $(4,5)$. 
Current prevalence of intraoperative injury of EBSLN has not been established in the literature so far because of the postoperative variability in voice symptoms and the difficulty of identifying the injury via the direct laryngoscopy and videostrobolaryngoscopy, compared with RLN injury (5). EBSLN injury rates have been reported to be approximately $58 \%$ (6). Thus, EBSLN injury is the most underestimated complication after thyroid surgery (5).

Several surgical techniques have been described to preserve EBSLN during the dissection of the superior thyroid pole, but none of them has been determined to be superior enough to decrease the potential risk of EBSLN injury (5). Intraoperative neuromonitoring (IONM) has gained widespread acceptance as an adjunct to the gold standard of visual identification of RLN (7). Routine identification of EBSLN was thought to be almost impossible and harmful to the nerve previously. However, IONM has recently been reported to be helpful and less invasive for identifying EBSLN (8-11). As these findings increased the surgical interest in using neuromonitoring for the identification of EBSLN, the rate of neuromonitoring use has increased over time. On an international survey in surgeons with known interest in thyroid surgery, the International Neural Monitoring Study Group in Thyroid and Parathyroid Surgery reported that IONM was used for the identification of the EBSLN by $26.3 \%$ of low-volume versus $68.4 \%$ of high-volume surgeons $(p=0.004)$ worldwide, and $93 \%$ of respondents believed that EBSLN identification with IONM is necessary for voice professionals (12). We have been using IONM for EBSLN exploration since July 2012. In the current study, we aimed to evaluate the contribution of IONM to visual and functional identification of EBSLN.

\section{MATERIAL AND METHODS}

The prospectively collected data of 126 (female, 103; male, 23) patients undergoing thyroid surgery via IONM for the EBSLN exploration from January to December 2015 were assessed retrospectively. Each side of the neck was accepted as a separate entity, and 215 neck sides were assessed.

Patients were informed of the intent of using a monitoring system to potentially aid in avoiding any possible injury to RLN and EBSLN and assessing their functions during the operation. The patients provided written informed consent for the surgical procedure as well as the study and publication of the stored results according to the institutional review board. This study was approved by the hospital's Ethics Committee.

The exclusion criteria were revision surgery, thyroid cancer with massive extrathyroidal extension, intentional nerve transection because of cancer invasion, and failure to assess EBSLN function because of technical issues.

Medical records were collected and stored within a prospective database. The collected information included demographic features, indications for thyroid surgery, operative details (such as type of surgery and the IONM data) and postoperative period details.

\section{Intraoperative Neuromonitoring Technique}

The NIM-3.0 Neuro Monitoring System (Medtronic Xomed; Jacksonville, FL, USA) with endotracheal surface electrodes was under general anesthesia with a low-dose short-acting neuromuscular blockade (rocuronium $0.3 \mathrm{mg} / \mathrm{kg}$ ) and intubated with Medtronic Xomed Nerve Integrity Monitor Standard Reinforced Electromyography Endotracheal Tube (size 6.0, 7.0, or 8.0 Fr). A sterile, single-use, pulse-generated monopolar stimulator probe (Medtronic Xomed; Jacksonville, FL, USA) was applied for nerve stimulation in the operative field with an interrupted stimulation technique, where in the probe was set to a value of $1 \mathrm{~mA}$, impulse duration at $100 \mu \mathrm{s}$, frequency at 4 $\mathrm{Hz}$, and the monitor was set for an event threshold of $100 \mu \mathrm{V}$. IONM setup, applications, and data interpretation were consistent with the International Neural Monitoring Guidelines $(5,7)$. The electromyographic waveform amplitude defined the vocal cord adductor function. Standard IONM was carried out as a 4-step procedure for RLN (V1, R1, R2, and V2). EBSLN function was evaluated via CTM twitch. An auditory signal and obvious glottis electromyographic waveform with EBSLN stimulation were also obtained in some cases and these electromyographic responses were recorded. Positive stimulation was defined as both the audible alarm of the monitoring system and achievement of a recognizable electromyographic waveform amplitude that was $>100 \mu \mathrm{V}$, for both RLN and EBSLN.

\section{Surgical Technique}

Thyroidectomy and/or central neck dissection was performed by a 4-6-cm collar transverse incision. Subplatysmal flaps were raised, strap muscles were divided along the midline, the thyroid lobe was medialized, and middle thyroid vein was divided. A previously described systematic approach is used consistently for monitoring and identification of RLNs (13).

Superior thyroid pole dissection: Superior pole dissection was performed before or after lateral gland mobilization. The upper pole of the lobe was retracted laterally and caudally to expand the sternothyroid-laryngeal triangle (SLT). In cases of enlarged gland or short neck, the strap muscles were transected with ligasure (Covidien, Mansfield, MA) at one-third of superior edge with care to preserve the ansa cervicalis to provide a better exposure and dissection of the superior thyroid pedicle $(5,14)$.The avascular space between the superior pole and CTM was dissected bluntly for a better exposure of the SLT where the EBSLN lies. After the visual identification of EBSLN in the SLT, confirmation analysis with a monopolar stimulator probe via CTM twitch assessment was performed. If the EBSLN was not identified visually, it was searched with a stimulator probe under the laryngeal head of the sternothyroid muscle, which is a good landmark for the EBSLN's course, and followed over the inferior pharyngeal constrictor (IPC) muscle down towards the CTM (15). When EBSLN was not able to be identified using a probe, the fibers of IPC muscle were not dissected through to identify the nerve visually.

While managing the superior pole dissection, each portion of the pedicle tissue was stimulated with a probe to make sure that EBSLN is not trapped during the manipulations of the surgeon. After dividing the superior thyroid vessels, we stimulated EBSLN with a probe cranially to the division point of the vessels to confirm the integrity of EBSLN.

\section{Evaluation of Vocal Cord Function}

Patients underwent a routine pre- and postoperative (within 2 days) direct laryngoscopy, directed by an independent laryngol- 
Table 1. Demographic profile, indications, and types of surgery

\begin{tabular}{|lc|}
\hline Variable & $\mathbf{n}$ \\
\hline Gender (Female/Male) & $103 / 23$ \\
\hline Mean age (year \pm SD) & $46.2 \pm 12.2$ \\
\hline Range & $(18-75)$ \\
\hline Preoperative diagnosis & \\
\hline Benign thyroid disease & 83 \\
$\quad$ euthyroid & 65 \\
\hline \multicolumn{1}{|c|}{ hyperthyroid } & 18 \\
\hline Suspicious malignancy & 21 \\
\hline Malignant & 22 \\
\hline Procedure & \\
\hline
\end{tabular}

\begin{tabular}{|l|l|}
\hline Bilateral approach & \\
\hline TT & 70 \\
\hline TT+CLND & 15 \\
\hline TT+CLND+LND & 4 \\
\hline Unilateral approach & 37 \\
\hline \multicolumn{2}{|l|}{ Lobectomy } \\
\hline EBSLNs at risk & 215 \\
\hline RLNs at risk & 215 \\
\hline
\end{tabular}

SD: standard deviation; TT: total thyroidectomy; CLND: central lymph node dissection; LD: lateral dissection; RLN: recurrent laryngeal nerve; EBSLN: external branch of the superior laryngeal nerve

Table 2. The distribution of EBSLN identification methods

\begin{tabular}{|lc|}
\hline EBSLN identification methods & $\mathrm{n}(\%)$ \\
\hline \begin{tabular}{l} 
Visual identification \\
\multicolumn{1}{|l}{$\begin{array}{l}\text { Direct visual identification } \\
\text { Visualization after identification with probe }\end{array}$}
\end{tabular} & $80(27.9)$ \\
\hline $\begin{array}{l}\text { Not visualized } \\
\quad \text { Identification with probe, (functional } \\
\text { identification) }\end{array}$ & $52(24.1)$ \\
\hline $\begin{array}{l}\text { Total identification } \\
\text { Not identified }\end{array}$ & $201(93.5)$ \\
\hline Total EBSLN at risk & $14(6.5)$ \\
\hline EBSLN paresis (temporary) & $(93.5)$ \\
\hline EBSLN: external branch of the superior laryngeal nerve & $3(1.5)$ \\
\hline
\end{tabular}

ogist. RLN palsy was determined to be permanent when there was no evidence of recovery within 6 months after the surgery.

The visual identification rate of EBSLN and IONM's contribution to the visual and functional identification of EBSLN were evaluated.

\section{Statistical Analyses}

The incidence of nerve events was calculated based on the number of nerves at risk. Differences between continuous and categorical variables were assessed by the Mann-Whitney $U$ test and Fisher's exact test or chi-square test, respectively. A value of $p<0.05$ was considered to be statistically significant.

\section{RESULTS}

The study includes the data of 126 patients. Demographic characteristics, indication for surgery, and types of surgical procedures performed are presented in Table 1.

The methods used for EBSLN identification are described in Table 2. Two hundred and one (93.5\%) of 215 EBSLNs were identified, but 14 (6.5\%) EBSLNs were not able to be identified with IONM. Sixty (27.9\%) EBSLNs were visually identified before being stimulated with a monopolar stimulator probe. Eighty-nine (41.4\%) EBSLNs were visually identified after being stimulated with a probe. Although 52 (24.1\%) EBSLNs were identified with a probe, they were not visualized. The IONM provided a significant contribution to visual $(p<0.001)$ and functional $(p<0.001)$ identification of EBSLN.

Additionally, positive electromyographic responses were recorded from 160 EBSLNs (74.4\%). The amplitudes of the electromyographic waveforms obtained from RLNs and EBSLNs were $865 \pm 607 \mu \mathrm{V}$ (range, 311-2770) (Figure 1a) and $181 \pm 92 \mu \mathrm{V}$ (range, 100-392) (Figure 1b), respectively, with a significant difference when compared with each other $(p<0.001)$. However, no CTM twitch was observed via stimulating the proximal parts of the three (1.5\%) of $201 \mathrm{EBSLNs}$, although they were visually intact at the end of the surgery (Table 2). Voice changes were detected in three patients with identified EBSLNs, who had loss of signal on the proximal parts of EBSLNs at the end of their operations. Voice complaints of these three patients, such as inability to produce high-pitched sounds and vocal fatigue at the end of the day, improved within 4 months. There was no vocal cord paralysis in these patients.

All the vocal cord paralyses detected were unilateral. 11 (5.1\%) temporary and $1(0.5 \%)$ permanent RLN paralyses (RLNP) were detected.

\section{DISCUSSION}

External branch of the superior laryngeal nerve is in close relation with the superior thyroid vessels, causing it to be at risk during thyroid surgery. Various anatomical classification systems for EBSLN have been proposed including the Cernea, Friedman, and Kierner classifications (5). Cernea classification describes EBSLN to have three types with regard to its relation with superior thyroid vessels and superior thyroid pole.Type 1 EBSLN crosses the superior thyroid vessels more than $1 \mathrm{~cm}$ from the upper edge of the superior pole, while Type $2 \mathrm{~A}$ nerve crosses the vessels less than $1 \mathrm{~cm}$ above the upper edge of the superior pole. Type $2 \mathrm{~B}$ nerve crosses the superior thyroid vessels below the upper edge of the superior thyroid pole. This classification is the most widely accepted one that is based on the potential risk of injury to EBSLN during thyroid surgery (16). The rate of EBSLNs according to the Cernea classification has been reported in studies with results varying from $17 \%$ to $60 \%$ for Type $1,17 \%$ to $59 \%$ for Type $2 A$, and $10 \%$ to $56 \%$ for Type $2 B(4,8,9,17,18)$. The different results may be due to various factors such as, the race of the individual, height of the individual, weight of the thyroid gland, identification type of EBSLN (visually or with the use of IONM), and the extension of the study. Both Type $2 \mathrm{~A}$ and $2 \mathrm{~B}$, but mostly Type $2 \mathrm{~B}$, EBSLNs 
a

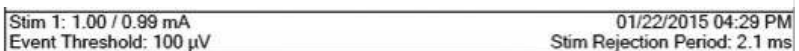

Event Threshold: $100 \mu \mathrm{V}$ Stim Rejection Period: $2.1 \mathrm{~ms}$

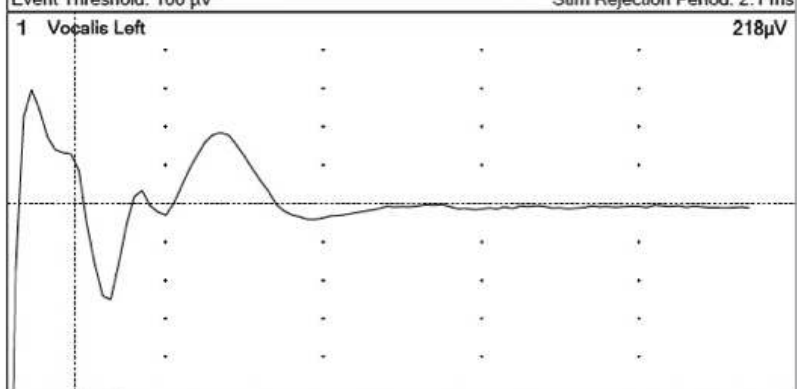

2 Voçalis Right $919 \mu \mathrm{V}$

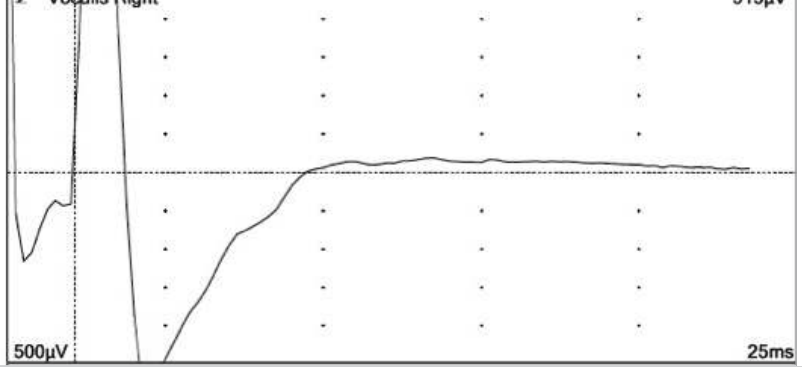

b

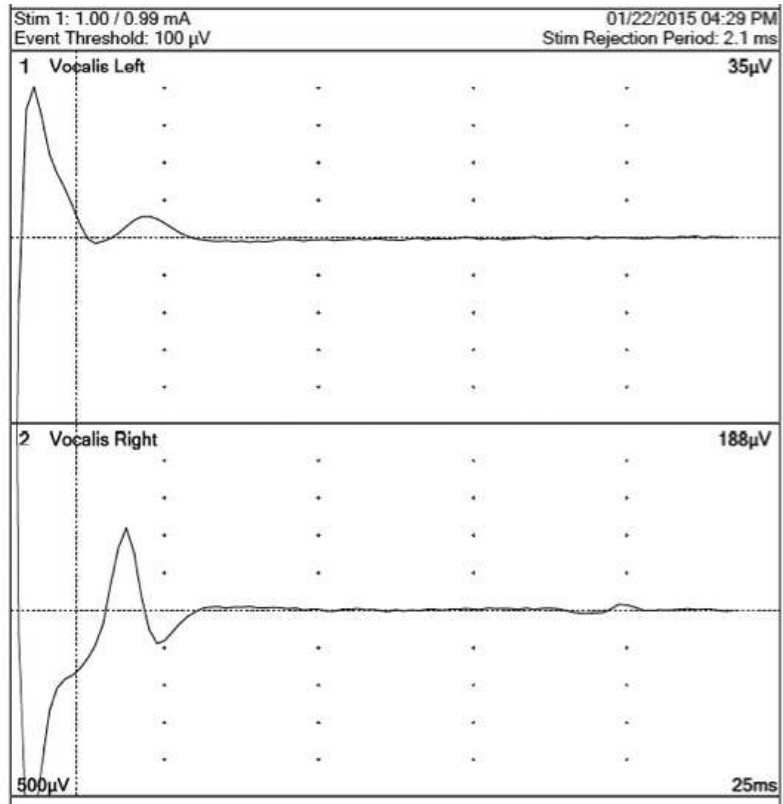

Figure 1. a, b. (a) The electromyographic waveform, with an amplitude of $919 \mu \mathrm{V}$, recorded from the right vocal cord via right RLN stimulation at the end of lobectomy. (b) The electromyographic waveform, with an amplitude of $188 \mu \mathrm{V}$, recorded from the right vocal cord via right EBSLN stimulation in the same patient

are at an increased risk of injury due to their close course to the superior thyroid vessels $(4,5)$.

Physical and functional preservation of EBSLN is as important as that of RLN and parathyroid glands for a safe thyroid surgery. Although the visual identification of RLN is accepted to be the gold standard to avoid injury, there is still no standard technique to preserve EBSLN and no consensus on the surgical protocol for EBSLN identification (19). Three techniques have been described to avoid EBSLN injury during the superior pole dissection. The first technique is the peripheral ligation of the individual branches of the superior thyroid vessels just on the thyroid capsule. The second technique is the visual nerve identification before ligating the vessels. The last one is the identification of the nerve with either a nerve stimulator or IONM before ligating the superior thyroid pole vessels $(4,5,10,20)$.

Some surgeons' expression that "I avoid injuring EBSLN by presuming where it should be, but I have never seen it" is not an appropriate approach for modern surgical principles. The fundamental principle of any surgery is the identification of a structure to avoid injuring it (21). Although nearly all RLNs can be visually identified, approximately $20 \%$ of the EBSLNs can not be visually identified due to their subfascial/ intramuscular course into inferior constrictor muscle, which makes it necessary to conduct a microdissection for the identification of the nerve (22). Similarly, $25.2 \%$ of all the EBSLNs were identified with IONM, but all of them were not able to be visualized in the present study. In the present study, the visual identification rate of EBSLNs before being stimulated with a probe was only $27.9 \%$, which was lower than the rate of $33 \%-93 \%$ reported in other studies $(2,10,11,17,20)$. In our opinion, the reason for this low rate is that we did not divide the strap muscles to expose the sternothyroid-laryngeal triangle, except only in the patients with enlarged thyroid lobes and a short neck. EBSLN identification rate was $34.1 \%$ in another study similar to ours in which the sternothyroid muscles were not divided, but the rate was high as $80 \%-88 \%$ in other studies where the sternothyroid muscles were divided to improve visual control of EBSLN $(8-10,17)$. However, the visual identification rate of EBSLN increased to $68.3 \%$ with the aid of IONM, which is close to the rates reported in the studies that included the routine division of strap muscles during superior pole dissection. In this study, the functional identification rate of EBSLN with IONM was noted to be $93.5 \%$, which is threefold of the visual identification rate and in accordance with the rate of $83 \%-100 \%$ reported in other studies $(2,8,10$, $11,15,23)$. We demonstrated that IONM provided a meaningful contribution to visual $(p<0.001)$ and functional $(p<0.001)$ identification of EBSLN. Additionally, it is also important to verify the visually identified structure by IONM because nonneural fibers or tendinous fibers of regional muscles can be confused for EBSLN (23). It is not always possible to identify EBSLN even for experienced thyroid surgeons. EBSLN is very fine, about $0.8 \mathrm{~mm}$ in diameter, and often invisible because of its course through the pharyngeal constrictor muscle (2). In cases in which surgeons cannot identify EBSLN, they can only assume that it is not injured with ligation of small portions of superior thyroid vessels (2).The widespread use of thermal sealing devices is another threatening factor for the preservation of the nerve (24). EBSLN's course can be only affirmed with the use of $\operatorname{IONM}(5,10,22)$.

The amplitude of the electromyographic waveform defined the adductor motor function of the vocal cords, whose main adductor is the thyroarytenoid muscle. In addition to the observed CTM twitch, electromyographic data could be achieved with EBSLN stimulation via the use of standard monitoring tubes in $70 \%-80 \%$ of cases $(10,25,26)$. The distant most fibers of EBSLN penetrate the two heads of CTM extend- 
ing to the anterior portion of thethyroarytenoid muscle and connect with RLN. This connection has been described as the "human communicating nerve" and is known to contribute to the motor innervation of the thyroarytenoid muscle $(25,27)$. The mean amplitude of EBSLN stimulation was significantly lower than that of $\operatorname{RLN}(10,15,25)$. Similar to these previous studies, we also achieved electromyographic responses from $74.4 \%$ of EBSLNs identified by IONM and the mean amplitude was $181 \pm 92 \mu \mathrm{V}$, which is lower than that of RLN $(865 \pm 607 \mu \mathrm{V})$. Darr et al. (15) could find all EBSLNs and obtained recognizable electromyographic responses in $100 \%$ of cases via the use of a novel endotracheal tube having electrodes with an extended surface area, in their prospective study. They found that the electromyographic responses from the vocal cords had a great variability and tube position might influence the electromyographic waveform amplitudes. The mean electromyographic amplitudes were found to be similar between genders. Multiple nerve stimulations and extensive dissection did not affect EBSLN amplitude (15).

The reported prevalence of EBSLN injury alters widely from $0 \%$ to $58 \%(4,6,8,10,28)$. It is difficult to evaluate the actual prevalence due to the limited data, difficulty of quantifying EBSLN function, and differences in diagnostic techniques in various studies. CTM electromyography is the only definitive way to detect EBSLN injury because of the variability in vocal symptoms and findings at the postoperative laryngeal examination (5). Electromyography of CTM is too invasive to perform routinely and can be difficult to comment on secondary to variability in electromyographic needle placement. It is, therefore, very important to confirm that EBSLN is preserved during thyroidectomy by IONM (2).

\section{Limitations of the Study}

The present study has several limitations. First, the study is a retrospective one, although the data were collected prospectively. The patients without vocal cord paralysis and loss of signal in EBSLNs did not have voice change complaints. All three patients with identified EBSLNs via IONM had loss of signal on the proximal parts of EBSLNs after the dissection, supporting the fact that the voice change complaints were due to EBSLN injury. The voice change complaints of the patients were all temporary. However, one of the major limitations is that we could not evaluate the exact prevalence of EBSLN injuries in the patients postoperatively by electromyography. It is not a practical method to apply to all the patients who undergo thyroidectomies.

\section{CONCLUSION}

As a conclusion, IONM provides a significant contribution to the preservation of EBSLN by improving both the visual and functional identification of it. We think that EBSLN risk is unpredictable and IONM should be used in the superior pole dissection routinely. Although EBSLN's main function is to innervate CTM, it also contributes to the vocal cord's adductor function due to the presence of communicating nerves between RLN and EBSLN. Thus, glottis electromyographic response obtained with EBSLN stimulation provides quantifiable information in addition to the simple visualization of CTM twitch.
Ethics Committee Approval: Ethics committee approval was received for this study from the ethics committee of Şişli Hamidiye Etfal Training and Research Hospital.

Informed Consent: Written informed consent was obtained from patients who participated in this study.

Peer-review: Externally peer-reviewed.

Author Contributions: Concept - N.A., M.U.; Design - N.A.; Supervision - M.U., A.I.; Resource - N.A., M.U; Materials - N.A.; Data Collection and/or Processing - N.A.; Analysis and/or Interpretation - N.A., M.U.; Literature Search - N.A., M.U.; Writing Manuscript - N.A., M.U.; Critical Reviews M.U., A.I.;

Conflict of Interest: No conflict of interest was declared by the authors.

Financial Disclosure: The authors declared that this study has received no financial support.

\section{REFERENCES}

1. Henry LR, Helou LB, Solomon NP, Howard RS, Gurewich-Uvena J, Coppit G, et al. Functional voice outcomes after thyroidectomy: an assessment of the Dsyphonia Severity Index (DSI) after thyroidectomy. Surgery 2010; 147: 861-870. [CrossRef]

2. Masuoka H, Miyauchi A, Higashiyama T, Yabuta T, Fukushima M, Ito $Y$, et al. Prospective randomized study on injury of the external branch of the superior laryngeal nerve during thyroidectomy comparing intraoperative nerve monitoring and a conventional technique. Head Neck 2015; 37: 1456-1460. [CrossRef]

3. Sanders I, Wu BL, Mu L, Li Y, Biller HF. The innervation of the human larynx. Arch Otolaryngol Head Neck Surg 1993; 119: 934939. [CrossRef]

4. Cernea CR, Ferraz AR, Furlani J, Monteiro S, Nishio S, Hojaij FC, et al. Identification of the external branch of the superior laryngeal nerve during thyroidectomy. Am J Surg 1992; 164: 634-639. [CrossRef]

5. Barczyński M, Randolph GW, Cernea CR, Dralle H, Dionigi G, Alesina $P F$, et al. External branch of the superior laryngeal nerve monitoring during thyroid and parathyroid surgery: International Neural Monitoring Study Group standards guideline statement. Laryngoscope 2013; 123 Suppl 4: S1-14. [CrossRef]

6. Jansson S, Tisell LE, Hagne I, Sanner E, Stenborg R, Svensson P. Partial superior laryngeal nerve (SLN) lesions before and after thyroid surgery. World J Surg 1988; 12: 522-527. [CrossRef]

7. Randolph GW, Dralle $\mathrm{H}$; International Intraoperative Monitoring Study Group, Abdullah H, Barczynski M, Bellantone R, Brauckhoff $M$, et al. Electrophysiologic recurrent laryngeal nerve monitoring during thyroid and parathyroid surgery: international standards guideline statement. Laryngoscope 2011; 121 Suppl 1: S1-16. [CrossRef]

8. Hurtado-López LM, Díaz-Hernández PI, Basurto-Kuba E, ZaldívarRamírez FR, Pulido-Cejudo A. Efficacy of intraoperative neuromonitoring to localize the external branch of the superior laryngeal nerve. Thyroid 2016; 26: 174-178. [CrossRef]

9. Glover AR, Norlén O, Gundara JS, Morris M, Sidhu SB. Use of the nerve integrity monitor during thyroid surgery aids identification of the external branch of the superior laryngeal nerve. Ann Surg Oncol 2015; 22: 1768-1773. [CrossRef]

10. Barczyński M, Konturek A, Stopa M, Honowska A, Nowak W. Randomized controlled trial of visualization versus neuromonitoring of the external branch of the superior laryngeal nerve during thyroidectomy. World J Surg 2012; 36: 1340-1347. [CrossRef]

11. Dionigi G, Boni L, Rovera F, Bacuzzi A, Dionigi R. Neuromonitoring and video-assisted thyroidectomy: a prospective, randomized case-control evaluation. Surg Endosc 2009; 23: 996-1003. [CrossRef] 
12. Barczyński M, Randolph GW, Cernea C; International Neural Monitoring Study Group in Thyroid and Parathyroid Surgery. International survey on the identification and neural monitoring of the EBSLN during thyroidectomy. Laryngoscope 2016; 126: 285-291. [CrossRef]

13. Uludag M, Aygun N, Isgor A. Motor function of the recurrent laryngeal nerve: Sometimes motor fibers are also located in the posterior branch. Surgery 2016; 160: 153-160. [CrossRef]

14. O'Neill CJ, Chang LY, Suliburk JW, Sidhu SB, Delbridge LW, SywakMS. Sutureless thyroidectomy: surgical technique. ANZ J Surg 2011; 81: 515-518. [CrossRef]

15. Darr EA, Tufano RP, Ozdemir S, Kamani D, Hurwitz S, Randolph G. Superior laryngeal nerve quantitative intraoperative monitoring is possible in all thyroid surgeries. Laryngoscope $2014 ; 124$ : 10351041. [CrossRef]

16. Cernea CR, Ferraz AR, Nishio S, Dutra A Jr, Hojaij FC, dos Santos LR. Surgical anatomy of the external branch of the superior laryngeal nerve. Head Neck 1992; 14: 380-383. [CrossRef]

17. Aina EN, Hisham AN. External laryngeal nerve in thyroid surgery: recognition and surgical implications.ANZ J Surg 2001; 71: 212214. [CrossRef]

18. Dionigi G, Kim HY, Randolph GW, Wu CW, Sun H, Liu X, et al. Prospective validation study of Cernea classification for predicting EMG alterations of the external branch of the superior laryngeal nerve. Surg Today 2016; 46: 785-791. [CrossRef]

19. Hermann M, Alk G, Roka R, Glaser K, Freissmuth M. Laryngeal recurrent nerve injury in surgery for benign thyroid diseases: effect of nerve dissection and impact of individual surgeon in more than 27,000 nerves at risk. Ann Surg 2002; 235: 261-268. [CrossRef]

20. Bellantone $R$, Boscherini $M$, Lombardi CP, Bossola M, Rubino F, De $\mathrm{Crea} C$, et al. Is the identification of the external branch of the superior laryngeal nerve mandatory in thyroid operation? Results of a prospective randomized study. Surgery 2001; 130: 10551059. [CrossRef]
21. Patnaik U, Nilakantan A, Shrivastava T. Anatomical variations of the external branch of the superior laryngeal nerve in relation to the inferior constrictor muscle: cadaveric dissection study. J Laryngol Otol 2012; 126: 907-912. [CrossRef]

22. Lennquist $\mathrm{S}$, Cahlin $\mathrm{C}$, Smeds $\mathrm{S}$. The superior laryngeal nerve in thyroid surgery. Surgery 1987; 102: 999-1008.

23. Selvan B, Babu S, Paul MJ, Abraham D, Samuel P, Nair A. Mapping the compound muscle action potentials of cricothyroid muscle using electromyography in thyroid operations: a novel method to clinically type the external branch of the superior laryngeal nerve. Ann Surg 2009; 250: 293-300. [CrossRef]

24. Dionigi G. Energy based devices and recurrent laryngeal nerve injury: the need for safer instruments. Langenbecks Arch Surg 2009; 394: 579-580; author reply 581-586. [CrossRef]

25. Potenza AS, Phelan EA, Cernea CR, Slough CM, Kamani DV, Darr $A$, et al. Normative intra-operative electrophysiologic waveform analysis of superior laryngeal nerve external branch and recurrent laryngeal nerve in patients undergoing thyroid surgery. World J Surg 2013; 37: [CrossRef]

26. Aygün N, Besler E, Celayir F, Bozdağ E, Çitgez B, Yetkin G, et al. The effect of the intraoperative neuromonitoring to the external branch of the superior laryngeal nerve identification and contribution of the nerve to the motor function of the thyroaritenoid muscle. ŞEHTB 2016; 50: 97-102.

27. $\mathrm{Wu} \mathrm{Bl}$, Sanders I, Mu L, Biller HF. The human communicating nerve.An extension of the external superior laryngeal nerve that innervates the vocal cord. Arch Otolaryngol Head Neck Surg 1994; 120: 1321-1328. [CrossRef]

28. Hurtado-López LM, Pacheco-Alvarez MI, Montes-Castillo Mde L, Zaldívar-Ramírez FR. Importance of the intraoperative identification of the external branch of the superior laryngeal nerve during thyroidectomy: electromyographic evaluation. Thyroid 2005; 15: 449-454. [CrossRef] 